\title{
Bifidogenic effect and stimulation of short chain fatty acid production in human faecal slurry cultures by oligosaccharides derived from lactose and lactulose
}

\author{
Alejandra Cardelle-Cobas ${ }^{1}$, María Fernández ${ }^{2}$, Nuria Salazar ${ }^{2}$, Cristina Martínez-Villaluenga ${ }^{1}$, \\ Mar Villamiel $^{1 *}$, Patricia Ruas-Madiedo ${ }^{2}$ and Clara G de los Reyes-Gavilán ${ }^{2}$ \\ ${ }^{1}$ Instituto de Fermentaciones Industriales (CSIC), Juan de la Cierva, 3, Madrid 28006, Spain \\ ${ }^{2}$ Instituto de Productos Lácteos de Asturias (CSIC), Crta. Infiesto s/n, 33300 Villaviciosa (Asturias), Spain
}

Received 11 August 2008; accepted for publication 9 January 2009; first published online 18 May 2009

Bifidogenic effect and stimulation of short chain fatty acid (SCFA) production by fractions of oligosaccharides with a DP $\geqslant 3$ and Gal $\beta(1-6)$ linkages synthesised from lactose or lactulose by Pectinex Ultra SP-L and Lactozym 3000 L HP G were evaluated in human faecal slurries. Results were compared with those obtained for the commercial oligosaccharide mixture Vivinal ${ }^{\circledR}$-GOS. Quantitative real-time PCR showed that all galacto-oligosaccharide (GOS) fractions stimulated slightly higher bifidobacteria growth than lactose, lactulose and Vivinal ${ }^{\circledR}$-GOS. GOS fractions promoted the production of total SCFA and acetic acid in a similar way to Vivinal ${ }^{\circledR}$-GOS and more than glucose, lactose and lactulose. In conclusion, oligosaccharides derived from lactose and lactulose may represent a suitable alternative to lactulose for the design of new functional food ingredients.

Keywords: Oligosaccharides, bifidogenic effect, short chain fatty acids, lactose, lactulose.

There is an increasing awareness of the importance of human intestinal microbiota in maintaining host health. Although little is known about all groups of bacteria involved in these beneficial properties, it is generally accepted that bifidobacteria and lactobacilli play a key role in this process (Tuohy et al. 2005).

Growth and metabolic activity of bifidobacteria can be selectively stimulated by non-digestible oligosaccharides considered as prebiotics (Gibson \& Roberfroid, 1995). At the same time prebiotics are able to reduce the populations of non-beneficial bacteria by favouring microbial competition (Chow, 2002). Short chain fatty acids (SCFA; acetate, propionate or butyrate) are the main end products arising from microbial fermentation of these carbohydrates and they are recognised to exert health benefits on the host (Cummings, 1981; Topping \& Clifton, 2001; Macfarlane \& Macfarlane, 2003). Enhanced SCFA production and increased delivery of these compounds in the distal colon, especially butyrate, may have a role in preventing colon cancer and other intestinal disorders (Wong et al. 2006).

Fermentation reactions in the colon are dependent on several factors related to the gut environment such as $\mathrm{pH}$ (Louis et al. 2007) and chemical composition of the

*For correspondence; e-mail: mvillamiel@ifi.csic.es available substrates (Wong et al. 2006). Thus, the relative resistance of oligosaccharides to microbial degradation mainly depends on the type of monosaccharide moieties, glycosidic linkages, and degree of polymerisation (DP) (Delzenne, 2003; Dongowski et al. 2005). In general, rapidly fermented oligosaccharides would show bifidogenic effects mainly in the caecum and proximal colon, whereas those more resistant would be able to modify the composition of the microbiota in the distal colon. Taking into account the complexity of the intestinal microbiota and that different areas of the colon may be affected by diseases, no single oligosaccharide preparation may be suitable for all applications or individuals (Holt et al. 2005).

Galactooligosaccharides (GOS) and lactulose are recognised as prebiotic carbohydrates and they are widely used in Europe, Japan and the United States (Tuohy et al. 2005). GOS are usually produced by enzymatic transgalactosylation of lactose (Mahoney, 1998; Panesar et al. 2006). Several commercial products containing GOS are currently available in the market, and they usually contain a mixture of tetrasaccharides, trisaccharides, lactose, glucose, and galactose (Shin \& Yang, 1998; Hsu et al. 2007).

Lactulose, a synthetic disaccharide manufactured by isomerization of lactose in basic media, was the first 
carbohydrate commercialised with recognised beneficial effects on gut bifidobacteria (Mendez \& Olano, 1979; Rycroft et al. 2001; Aider \& Halleux, 2007). However, its use can be limited since it is fermented in the proximal colon, which results in gas production (Cristl et al. 1992; Tuohy et al. 2005).

The understanding of structure-function relationships of prebiotics is directing the research towards specific structures with a higher level of functionality. In general, scarce information is currently available about the structurefunction relationships of these molecules (Barreteau et al. 2006). Recently, our research group has obtained mixtures of GOS derived from lactose and lactulose using commercial enzymatic preparations (Cardelle-Cobas et al. 2008a, b; Martínez-Villaluenga et al. 2008a, b). However, neither the bifidogenic effect of these oligosaccharides nor the production of compounds due to the metabolism of bifidobacteria has been tested. This information could be of great interest in order to broaden the range of compounds with improved functionality on the gut microbiota. In the present work, the stimulation of the bifidobacteria growth and metabolic activity of intestinal microbiota by oligosaccharides $(\mathrm{DP} \geqslant 3)$ derived from the reaction of lactose or lactulose with Pectinex-Ultra SP-L and Lactozym 3000 L HP G has been investigated in vitro by using human faecal slurry cultures.

\section{Materials and Methods}

\section{Reagents}

Lactose monohydrate and lactulose (>98\% purity) were supplied by Scharlau Chemie S.A. (Barcelona, Spain) and Fluka (Steinheim, Germany), respectively. Soluble enzymatic preparations of $\beta$-galactosidase from Aspergillus aculeatus (Pectinex Ultra SP-L) and Kluyveromyces lactis (Lactozym 3000 L HP G) were kindly provided by Novozymes A/S (Dittingen, Switzerland and Bagsvaerd, Denmark). Commercial product 'Vivinal ${ }^{\circledR}$-GOS' was a gift from Friesland Food Domo (Hanzeplein, The Netherlands), and contains approximately $45 \%$ GOS and $30 \%$ digestible sugars: lactose $(15 \%)$, glucose $(14 \%)$, and galactose $(1 \%)$ (Anthony et al. 2006). Bio-Gel P-2 fine grade with bead size $<45 \mu \mathrm{m}$ and molecular weight (MW) fractionation range 100-1,800 Da was purchased from Bio-Rad laboratories (Hemel Hempstead, Hertfordshire, U.K.).

\section{Enzymatic synthesis and purification of oligosaccharides derived from lactose and lactulose}

The enzymatic syntheses of GOS were carried out to obtain the highest yield of oligosaccharides with DP $\geqslant 3$. Four batches involving the combination of two substrates (lactose and lactulose) and two enzyme sources (Pectinex Ultra SP-L and Lactozym 3000 L HP G) were used as follows.
GOS-1 (Lactose-Lactozym derived oligosaccharides). A lactose solution of $250 \mathrm{~g} / \mathrm{l}$ was prepared in $0.05 \mathrm{M}$ potassium phosphate buffer containing $1 \mathrm{~mm} \mathrm{MgCl}_{2} \mathrm{pH} 7 \cdot 5$ and $\beta$-galactosidase from Lactozym $3000 \mathrm{~L} \mathrm{HP} \mathrm{G}$ was added at a final concentration of $3 \mathrm{U} / \mathrm{mL}$. Reaction was performed at $40{ }^{\circ} \mathrm{C}$ in an orbital shaker at $300 \mathrm{rpm}$ for $2 \mathrm{~h}$ (Martínez-Villaluenga et al. 2008a).

GOS-2 (Lactose-Pectinex derived oligosaccharides). Solutions of lactose $(300 \mathrm{~g} / \mathrm{L})$ were prepared in $0 \cdot 1 \mathrm{M}$-sodium phosphate buffer $\mathrm{pH}$ 6.5. $\beta$-galactosidase from Pectinex Ultra SP-L was added to a final concentration of $16 \mathrm{U} / \mathrm{ml}$. Reactions were performed at $60{ }^{\circ} \mathrm{C}$ in an orbital shaker at $300 \mathrm{rpm}$ for $7 \mathrm{~h}$ (Cardelle-Cobas et al. 2008a).

GOS-3 (Lactulose-Lactozym derived oligosaccharides). A lactulose solution of $250 \mathrm{~g} / \mathrm{l}$ was prepared in $0.05 \mathrm{M}$ potassium phosphate buffer containing $1 \mathrm{~mm}-\mathrm{MgCl}_{2} \mathrm{pH} 6.5$ and $\beta$-galactosidase from Lactozym $3000 \mathrm{~L} \mathrm{HP} \mathrm{G} \mathrm{was} \mathrm{ad-}$ ded at a final concentration of $6 \mathrm{U} / \mathrm{ml}$. Reaction was performed at $50{ }^{\circ} \mathrm{C}$ in an orbital shaker at $300 \mathrm{rpm}$ for $2 \mathrm{~h}$ (Martínez-Villaluenga et al. 2008b).

GOS-4 (Lactulose-Pectinex derived oligosaccharides). Solutions of lactulose $(450 \mathrm{~g} / \mathrm{l})$ were prepared in $0 \cdot 1 \mathrm{M}$ sodium phosphate buffer $\mathrm{pH} 6 \cdot 5$. $\beta$-galactosidase from Pectinex Ultra SP-L was added to a final concentration of $16 \mathrm{U} / \mathrm{ml}$. Reactions were performed at $60^{\circ} \mathrm{C}$ in an orbital shaker at $300 \mathrm{rpm}$ for $7 \mathrm{~h}$ (Cardelle-Cobas et al. 2008b).

Inactivation of enzymes was performed by immersion of reaction mixtures in boiling water for $5 \mathrm{~min}$. Mono- and disaccharides from the reaction mixtures as well as from the commercial Vivinal ${ }^{\circledR}$-GOS were removed using gel permeation chromatography. In brief, fractionation was performed in a column $(100 \times 2.5 \mathrm{~cm})$ of Bio-Gel P-2 (bead size $<45 \mu \mathrm{m}$ ) at $5{ }^{\circ} \mathrm{C}$. Bidistilled water was used as eluent at a flow rate of $15 \mathrm{ml} / \mathrm{h}$. Fractions of $5 \mathrm{ml}$ were collected in a fraction collector and the elution of carbohydrates was monitored by high-performance anionexchange chromatography with pulsed amperometric detection (HPAEC-PAD) as described later. Fractions of purified GOS containing a DP $\geqslant 3$ were pooled and freezedried for in vitro fermentation assays with human faecal slurries.

\section{Analysis of oligosaccharides by HPAEC-PAD}

GOS fractions were characterized by HPAEC-PAD on ICS2500 Dionex system consisting of GP50 gradient pump, and ED50 electrochemical detector with a gold working electrode and $\mathrm{Ag} / \mathrm{AgCl}$ reference electrode. Data acquisition and processing was performed with Chromeleon version 6.7 software (Dionex Corp., Sunnyvale, CA). For eluents preparation, MilliQ water (Millipore Ibérica, S.A.U., Madrid, Spain), $50 \%(\mathrm{w} / \mathrm{v}) \mathrm{NaOH}$ (Fluka, Germany) and 
$\mathrm{NaOAc}$ (Fluka, Germany) were used. All eluents were degassed by flushing with $\mathrm{He}$ for $25 \mathrm{~min}$. Separations were performed following the method described by Splechtna et al. (2006). Elution was at room temperature on a CarboPac PA-1 column $(250 \times 4 \mathrm{~mm})$ connected to a CarboPac PA-1 $(50 \times 4 \mathrm{~mm})$ guard column. Eluent A $(100 \mathrm{~mm}-\mathrm{NaOH})$, eluent $\mathrm{B}(100 \mathrm{~mm}-\mathrm{NaOH}$ and $50 \mathrm{~mm}-$ $\mathrm{NaOAc})$ and eluent $\mathrm{C}(100 \mathrm{~mm}-\mathrm{NaOH}$ and $1 \mathrm{~m}-\mathrm{NaOAc})$ were mixed to form the following gradient: $100 \% \mathrm{~A}$ from 0 to $20 \mathrm{~min}$ and 0 to $100 \% \mathrm{~B}$ from 20 to $70 \mathrm{~min}$. After each run, the column was washed for 10 min with $100 \%$ eluent $\mathrm{C}$ and re-equilibrated for $15 \mathrm{~min}$ with the starting conditions of the gradient used. Separations were performed at a flow rate of $1 \mathrm{ml} / \mathrm{min}$. Detection time and voltage parameters were set as follows: $\mathrm{E}_{1}=0 \cdot 1 \mathrm{~V}\left(\mathrm{t}_{1}=400 \mathrm{~ms}\right)$, $\mathrm{E}_{2}=-2.0 \mathrm{~V}\left(\mathrm{t}_{2}=10 \mathrm{~ms}\right), \mathrm{E}_{3}=0.6 \mathrm{~V}, \mathrm{E}_{4}=-0.1 \mathrm{~V}\left(\mathrm{t}_{4}=60 \mathrm{~ms}\right)$; $\mathrm{t}_{\mathrm{t}}=500 \mathrm{~ms}$. Samples and standard solutions were filtered through a $0.45 \mu \mathrm{m}$ nylon filter (Symta, Spain), $25 \mu \mathrm{l}$ were injected using an autosampler and separations were performed at a rate of $1 \mathrm{ml} / \mathrm{min}$. Quantification of oligosaccharides ( $D P \geqslant 3$ ) was performed by external calibration using raffinose (Sigma, St. Louis, MO) as standard.

\section{Faecal batch culture fermentations}

Three independent trials, each corresponding to faecal batch fermentations made in independent duplicate experiments by using samples of three different donors, were carried out in the carbohydrate-free basal medium (CFBM) described by Al-Tamimi et al. (2005). In each batch, CFBM was distributed into 8 glass tubes $(2.5 \mathrm{ml}$ per tube) containing each $15 \mathrm{mg}$ of different carbon sources: GOS mixtures derived from lactose and lactulose by different enzymatic reactions (GOS-1 to GOS-4) as well as Vivinal ${ }^{\circledR}-G O S$, and the reference sugars lactulose, lactose and glucose. One additional tube was kept without carbon source added and was used as a control. After complete dissolution, carbon sources were sterilized by filtration through a $0.45 \mu \mathrm{m}$ membrane. Before use, the tubes were maintained overnight at $37^{\circ} \mathrm{C}$ in anaerobiosis.

The faecal slurry inocula were prepared as follows: faeces from three healthy adult volunteers (two female and one male, 30-40 years old), who had not received antibiotics during the previous 6 months and had no history of gastrointestinal disorder, were separately diluted in sterile $0 \cdot 17$ M-phosphate buffered saline (PBS) pH $7 \cdot 3$ to $10 \% \mathrm{w} / \mathrm{v}$ and homogenised with a Lab-Blender 400 stomacher (Seward Medical, London, UK) for $2 \mathrm{~min}$. The faecal homogenates of each donor $(10 \mathrm{ml})$ were poured into $90 \mathrm{ml}$ CFBM and allowed to stabilize by keeping at $37^{\circ}$ overnight $C$ under anaerobic conditions.

Control and tubes containing the different carbohydrates were mixed with $2.5 \mathrm{ml}$ stabilised faecal slurry, so that the final concentration of each carbohydrate was $0 \cdot 3 \%$. Fermentations were carried out at $37^{\circ} \mathrm{C}$ under anaerobic conditions $\left(10 \% \mathrm{v} / \mathrm{v} \mathrm{H}, 10 \% \mathrm{CO}_{2}\right.$, and $\left.80 \% \mathrm{~N}_{2}\right)$ in a chamber Mac 500 (Don Whitley Scientific, West
Yorkshire, UK). Samples were taken at 0,8 and $24 \mathrm{~h}$ of incubation. One $\mathrm{ml}$ of cultures were centrifuged $(12000 \mathrm{~g}$, $10 \mathrm{~min}$ ) at each time and pellets and supernatants were collected for analyses.

\section{Analysis of bifidobacteria in faecal cultures by quantitative real-time $P C R$}

The quantification of the Bifidobacterium population in faecal batch fermentations was performed by quantitative real-time PCR using previously described genus-specific primers (Gueimonde et al. 2004). DNA isolation was performed with the QIAamp ${ }^{\circledR}$ DNA stool Mini kit (Qiagen, $\mathrm{GmbH}$, Hilden, Germany) according to the instructions of the manufacturer.

All reactions were performed on MicroAmp optical plates sealed with MicroAmp optical caps (Applied Biosystems, Foster City, CA) and amplifications were carried out in a 7500 Fast Real Time PCR System (Applied Biosystems) using the SYBR Green PCR Master Mix (Applied Biosystems). One $\mu$ l purified DNA was used as template in the $25 \mu \mathrm{IPCR}$ reaction. Thermal cycling consisted of an initial cycle of $95{ }^{\circ} \mathrm{C} 10 \mathrm{~min}$ followed by 40 cycles of $95^{\circ} \mathrm{C} 15 \mathrm{~s}$ and $60^{\circ} \mathrm{C} 1 \mathrm{~min}$. Standard curves were made with the strain Bifidobacterium longum NCIMB8809 which was grown overnight in MRSC (MRS broth, Difco, Becton, Dickinson \& Co, France, Supplemented with $0.25 \%$ Cysteine-HCL, Sigma) under anaerobic conditions. Standard curves were obtained by plotting the cycle threshold $\left(C_{t}\right)$ values obtained for the standard culture as a linear function of the base-10 logarithm of the initial number of cells in the culture determined by plate counting. The numbers of Bifidobacterium cells in faecal samples was determined by comparing the $C_{t}$ values obtained with the standard curve. Samples were analysed in duplicate in at least two independent PCR runs for each faecal slurry trial.

\section{Analysis of SCFA in faecal batch cultures by GC}

The SCFA were quantified in supernatants of faecal cultures by GC-FID. Cell-free supernatants from faecal cultures were filtered through $0.2 \mu \mathrm{m}$ membranes and mixed with $1 / 10$ ethyl-butyric acid $(2 \mathrm{mg} / \mathrm{ml})$ as internal standard (IS) for checking reproducibility and for quantification. Samples were immediately analysed using a GC 6890N with a FID detector (Agilent Technologies Inc., Palo Alto, CA) coupled with an automatic injector 7683B Series (Agilent). Data were collected with the Agilent Chemstation software. Samples $(1 \mu \mathrm{l})$ were injected in the GC equipped with an HP-FFAP capillary column $(25 \mathrm{~m} \times 0.32 \mathrm{~mm}$ internal diameter, with a $0.52 \mu \mathrm{m}$ film thickness; Agilent) using $\mathrm{He}$ as gas carrier with a constant pressure of $8 \mathrm{psi}$. The temperature of the injector was kept at $220^{\circ} \mathrm{C}$ and the split ratio was 30:1. Chromatographic conditions were $130{ }^{\circ} \mathrm{C}$, $5 \mathrm{deg} \mathrm{C} / \mathrm{min}$ up to $180{ }^{\circ} \mathrm{C}$ and then $20 \mathrm{deg} \mathrm{C} / \mathrm{min}$ up to $220^{\circ} \mathrm{C}$ for column cleaning. The temperature of FID 

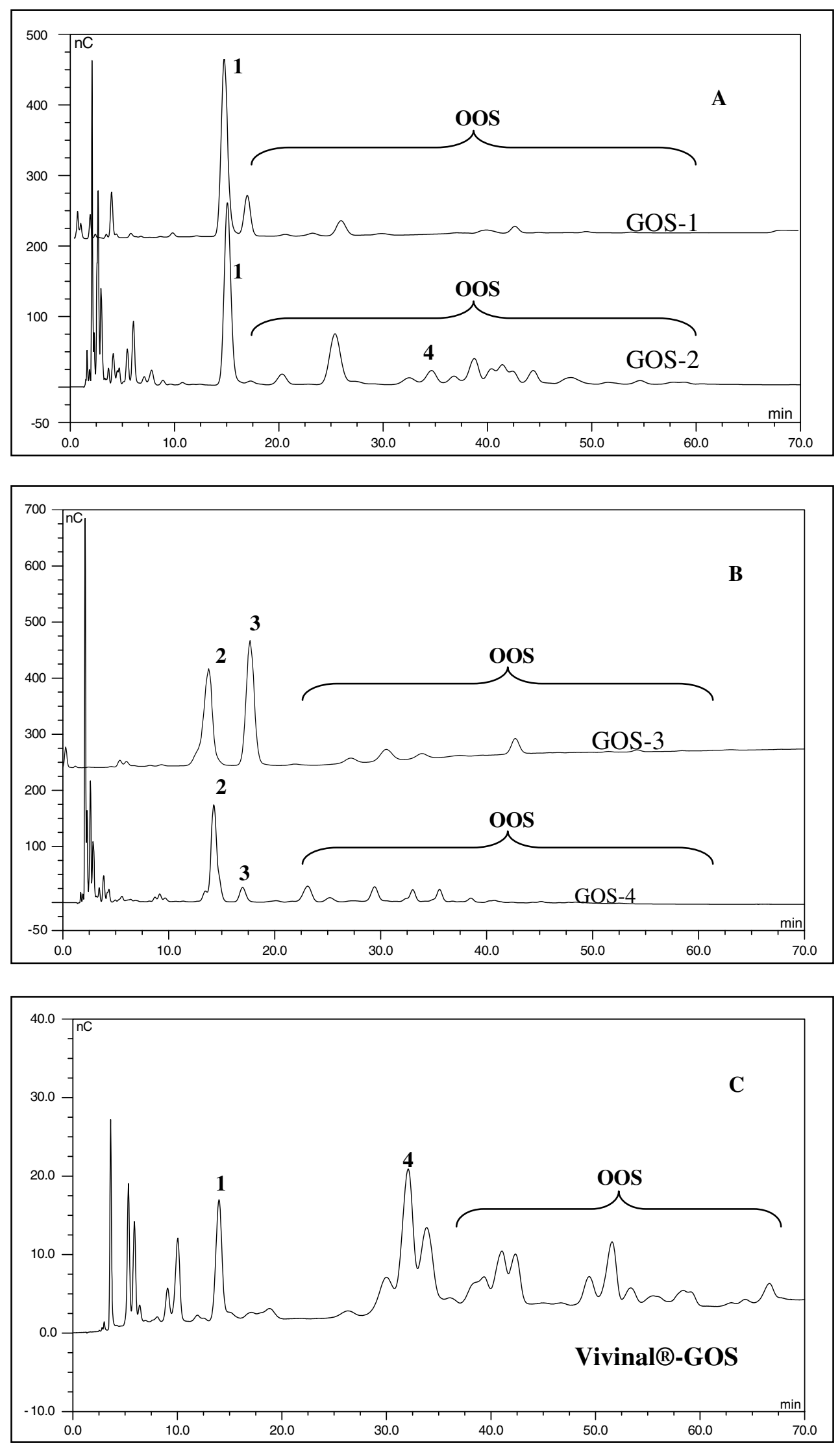

Fig. 1. For legend see opposite page. 
detector was kept at $250{ }^{\circ} \mathrm{C}$. The concentration $(\mathrm{ng} / \mathrm{ml})$ of each SCFA was calculated using the linear regression equations $\left(R^{2} \geqslant 0.99\right)$ from the corresponding curves of standards obtained with six different concentrations. Standards employed were acetic, propionic, isobutyric, butyric, isovaleric, valeric and caproic acids (Sigma Chemical Co., St. Louis, MO). Additionally, some samples were analysed by GC-MS to confirm their mass spectra in a GC $6890 \mathrm{~N}$ coupled to a mass spectrometer $5973 \mathrm{~N}$ detector (Agilent) employing the Enhanced ChemStation G1701DA software and the HP-Wiley 138 library.

\section{Results and Discussion}

\section{Chemical composition of oligosaccharide fractions derived from lactose and lactulose}

During the enzymatic hydrolysis of lactose and lactulose with Pectinex Ultra SP-L and Lactozym 3000 L HP G, complex mixtures of disaccharides and trisaccharides with linkages $\beta(1-6), \beta(1-4)$, and $\beta(1-3)$ are produced by transgalactosylation, trisaccharides being the main reaction products. However, considerable concentrations of monosaccharides as well as lactose, lactulose and other disaccharides remain in the reaction mixtures (Macfarlane et al. 2006; Cardelle-Cobas et al. 2008a, b; MartínezVillaluenga et al. 2008a, b). In order to obtain GOSenriched fractions, a purification step is needed. Figure 1 illustrates the chromatographic profiles obtained by HPAEC-PAD analysis for the purified oligosaccharide fractions derived from lactose $(\mathrm{A})$ and lactulose $(\mathrm{B})$ with Pectinex Ultra SP-L or Lactozym 3000L HP G and, moreover, the commercial mixture Vivinal ${ }^{\circledR}$-GOS (C) after purification as well. After purification, GOS-1 (LactoseLactozym derived oligosaccharides) fraction was enriched in $6^{\prime}$ galactosyl lactose (peak 1 ) being the major compound found $(81 \%)$. GOS-2 (Lactose-Pectinex derived oligosaccharides) fraction consisted of $42 \% 6^{\prime}$ galactosyl lactose (peak 1), 8\% 4' galactosyl lactose (peak 4) and $50 \%$ other oligosaccharides (OOS) with higher retention times. GOS-3 (Lactulose-Lactozym oligosaccharides) fraction was characterized mainly by the presence of two trisaccharides, 6' galactosyl lactulose (peak 2) and $\beta$-DGal-( $1 \rightarrow 4)$ - $\beta$-D-Fru- $(1 \rightarrow 1)-\beta$-D-Gal (peak 3 ) in a percentage of $36 \%$ and $47 \%$, respectively. GOS-4 (LactulosePectinex derived oligosaccharides) fraction exhibited $47 \%$ of $6^{\prime}$ galactosyl lactulose (peak 2$), 6 \%$ of $\beta$-D-Gal-( $1 \rightarrow 4$ )$\beta$-D-Fru- $(1 \rightarrow 1)-\beta$-D-Gal (peak 3 ) and $47 \%$ OOS with higher retention time. These compounds were identified by NMR (Martínez-Villaluenga et al. 2008b; Cardelle-Cobas et al. 2008b). As observed in Fig. 1, the chromatographic profile of the fractionated Vivinal ${ }^{\circledR}$-GOS is completely different from those obtained for oligosaccharides derived from lactose or lactulose with Pectinex Ultra SP-L and Lactozym $3000 \mathrm{~L} \mathrm{HP} \mathrm{G}$, since the former is a commercial product elaborated under different conditions and with other enzymes. The trisaccharide 4'-galactosyl-actose (peak 4) was purified and identified by NMR in the present work (data not shown). Chemical shifts of ${ }^{13} \mathrm{C}$ NMR spectra were coincident with those reported in the literature (Onishi et al. 1995). GOS mixture was composed of $24 \% 4^{\prime}$-galactosyl-lactose, 15\% 6' galactosyl lactose and $61 \%$ OOS. Chockchaisawasdee et al. (2005) reported than GOS fraction of Vivinal ${ }^{\circledR}$-GOS was composed of tri- and tetrasaccharides with a small amount of oligosaccharides with a higher DP which possessed a very high percentage of the 1,4 Gal linkage (55\% in the trisaccharide fraction and $72 \%$ in the higher oligosaccharide fraction).

\section{Changes of Bifidobacterium populations in faecal batch cultures analysed by quantitative real-time PCR}

Sugars such as glucose and lactose ingested with foods do not reach the colon because they are rapidly digested and adsorbed in the small intestine and, hence they cannot act as prebiotics. However, glycosidic linkages of lactulose and trisaccharides derived from lactulose and lactose make them more resistant to digestion (Tomomatsu, 1994), thus facilitating their arrival undegraded to the colon. Taking this consideration in mind, quantitative real-time PCR was used to evaluate the bifidogenic effect of the four synthesised GOS mixtures compared with the digestible sugars glucose and lactose as well as to the prebiotics lactulose and purified Vivinal ${ }^{\circledR}$-GOS (Fig. 2). In general, bifidobacteria populations increased through the incubation time, the highest increase being detected in cultures from individual 1 who presented the lowest initial bifidobacterial values. After 8 and $24 \mathrm{~h}$ of incubation all carbohydrate samples promoted higher increases of bifidobacteria populations than control cultures, with the exception of individual 3. In faecal cultures of this individual at $24 \mathrm{~h}$ of incubation the increase of counts in the control was of the same order as in the presence of the oligosaccharide samples. These results were indicative of a stimulatory effect on bifidobacteria growth by external carbon sources added in individuals 1 and 2 and also in individual 3 after $8 \mathrm{~h}$ of incubation. The degree of stimulation varied depending on the carbohydrate source and the individual, as it was previously stated for other carbohydrates by different authors. In general, the higher initial level of bifidobacteria

Fig. 1. HPAEC-PAD profiles of oligosaccharides with DP $\geqslant 3$ synthesised by Lactozym $3000 \mathrm{~L}$ HP G and Pectinex Ultra SP-L from lactose (GOS-1 and GOS-2, respectively) (A) or lactulose (GOS-3 and GOS-4, respectively) (B) and commercial Vivinal ${ }^{\circledR}-\mathrm{GOS}(\mathrm{C})$ obtained after purification with a Bio-Gel P-2 column. Peaks were identified as: (1) 6' galactosyl lactose, (2) 6' galactosyl lactulose, (3) $\beta$-D-Galp-(1 $\rightarrow 4)-\beta$-D-Fru-(1 $\rightarrow 1)-\beta$-D-Galp, (4) 4'-galactosyl lactose, OOS: other oligosaccharides. 

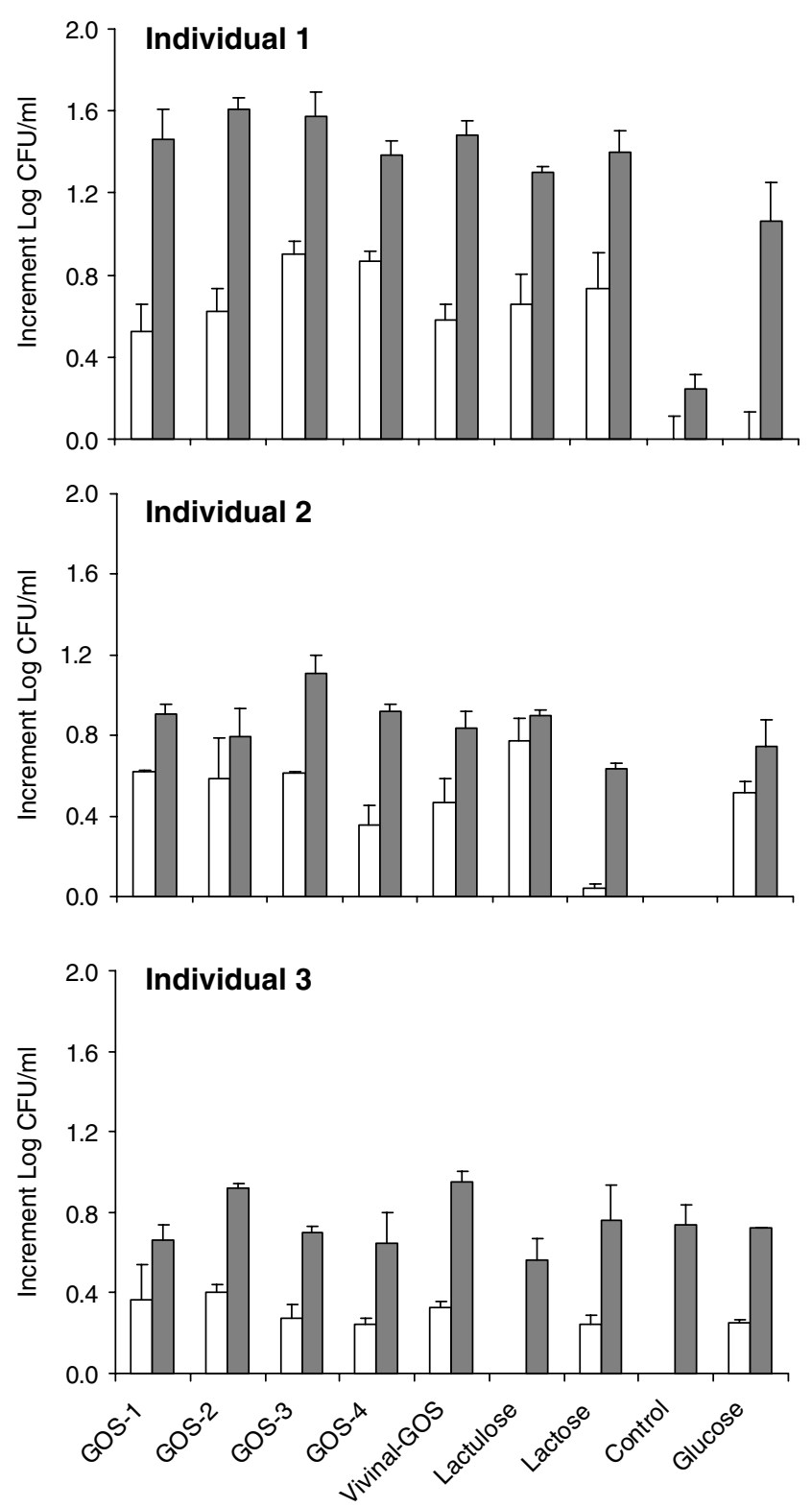

Fig. 2. Increase with respect to time 0 of Bifidobacterium counts measured by quantitative real-time PCR in faecal slurry cultures from three donors using as carbon sources purified GOS mixtures (obtained from lactose and lactulose), lactulose, Vivinal ${ }^{\circledR}$-GOS, lactose, or glucose after 8 (white bars) and $24 \mathrm{~h}$ (grey bars) of incubation. Control does not include carbohydrate source added. Initial Log CFU/ml Bifidobacterium counts: donor $1=8 \cdot 28 \pm 0 \cdot 13$, donor $2=8 \cdot 51 \pm 0 \cdot 02$, donor $3=8 \cdot 60 \pm 0 \cdot 05$. Error bars indicate standard deviation.

resulted in the lower increase of these microorganisms (Matteuzzi et al. 2004; De Preter et al. 2008; Salazar et al. 2008) and a great variability on stimulatory effect can be obtained, even when considering a numerous human population. In the present study, no noticeable differences were evidenced among the four GOS reaction mixtures within each individual. However, GOS obtained from lactose and lactulose promoted, in most cases, slightly higher count increases than the corresponding disaccharides from which they were derived. The bifidogenic effect of lactulose has been well established (Bouhnik et al. 2004a, b; Macfarlane et al. 2006; Vanhoutte et al. 2006). It is also known that chemical structures of oligosaccharides may affect their fermentation and prebiotic properties (Djouzi \& Andrieux, 1997; Rycroft et al. 2001). Thus, generally disaccharides with $\beta(1 \rightarrow 2), \beta(1 \rightarrow 4)$, and $\beta(1 \rightarrow 6)$ linkages are more suitable as prebiotics than disaccharides with other different linkages (Sanz et al. 2005) while no information was reported in relation to $\beta(1 \rightarrow 1)$ glycosidic linkages. Moreover, in general, carbohydrates with DP $=3$ showed the highest selectivity towards bifidobacteria (Kaneko et al. 1994; Kaplan \& Hutkins, 2000) which agreed with our results. In this way our results clearly indicated that GOS mixtures derived from lactose and lactulose which contain in their composition trisaccharides with high percentage of Gal $\beta(1 \rightarrow 6)$ and/or $\beta(1 \rightarrow 1)$ linkages, exhibited a bifidogenic effect similar or even slightly higher than their precursor disaccharides and the commercial oligosaccharide Vivinal ${ }^{\circledR}$-GOS. Further studies targeting other intestinal microbial groups such as beneficial bacteria, commensal microorganisms and opportunistic pathogens could help to establish the possible prebiotic role of these new GOS mixtures.

\section{Production of SCFA in faecal batch cultures analysed by GC-MS}

In general, the increase of total SCFA after 8 and $24 \mathrm{~h}$ incubation was more pronounced in samples with carbohydrates added than in faecal cultures without any external carbon source, thus indicating that all carbohydrates tested were able to act as fermentable substrates for the intestinal microbiota, clearly enhancing the production of SCFA during incubation. Differences between individuals were evident with respect to the levels of SCFA finally attained after incubation, the amount of total SCFA produced following the order individual $1>$ individual $2>$ individual 3 (Table 1). Thus, whereas the increase of both propionic and butyric acids was moderate, a clear rise of acetic acid occurred during incubation. This increase in acetic acid was directly related to the increase of bifidobacteria counts in faecal cultures determined by quantitative real-time PCR (Table 1 \& Fig. 2). As a consequence of this behaviour, a clear rise of the acetic to propionic acids ratio was found during incubation of faecal cultures from individuals 1 and 2. In this respect, several authors stated the enhancement of SCFA production by lactulose and other prebiotics using in vitro and in vivo models (Macfarlane et al. 2006). Rycroft et al. (2001) highlighted the capacity of lactulose and GOS to give rise to higher concentrations of acetic and total SCFA than other oligosaccharides after short-term incubations.

With respect to the influence of the type of carbohydrate tested on SCFA production no differences were 
Table 1. Concentrations $(\mathrm{mm})$ of the three major SCFA in faecal cultures from three donors using purified GOS mixtures derived from lactose and lactulose, Vivinal ${ }^{\circledR}-G O S$, lactulose, lactose and glucose as carbon sources

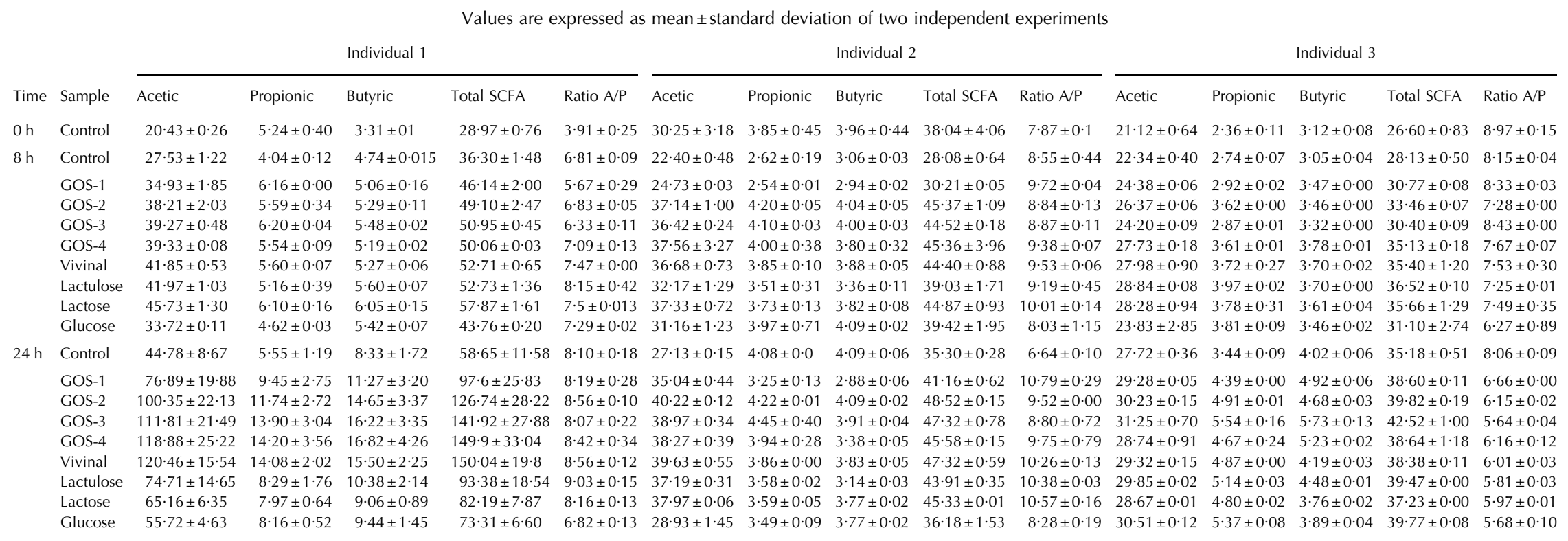

GOS-1: lactose-Lactozym derived oligosaccharides, GOS-2: lactose-Pectinex derived oligosaccharides, GOS-3: lactulose-Lactozym derived oligosaccharides, GOS-4: lactulose-Pectinex derived oligosaccharides 
found among carbohydrates in cultures from individual 3, who was the lowest SCFA producer. GOS-2, GOS-3, and GOS-4 mixtures were the best substrates for SCFA production, reaching levels of acetic acid and total SCFA similar to Vivinal ${ }^{\circledR}$-GOS and moderately higher than lactulose, mainly for samples from individual 1 who was the strongest SCFA producer. In spite of the different origins and methods employed of obtaining lactulose, Vivinal ${ }^{\circledR}$ GOS and GOS mixtures, no conclusion can be reached on a possible relationship between the degree of efficiency as bifidogenic or fermentable substrates of trisaccharides identified in the mixtures and their glycosidic linkages and composition. The moderately higher production of SCFA in the presence of our GOS fractions with respect to lactulose could be attributed to the synergistic effect of the different compounds present in the GOS mixtures.

In short, the results presented here allow a preliminary comparison to be made of the bifidogenic properties of different types of GOS including new lactulose derived oligosaccharides. The four purified fractions derived from lactose and lactulose act as fermentable substrates for intestinal bacteria in vitro, enhancing the production of acetic acid and SCFA and promoting the increase of Bifidobacterium numbers in human faecal cultures. To the best of our knowledge, this is the first study of the bifidogenic effect of the oligosaccharides derived from lactulose. The GOS derived from lactose and, specially, the novel oligosaccharides derived from lactulose may represent a suitable alternative to lactulose for the design of new functional food ingredients. Further in vivo studies using animal models and human volunteer trials are necessary in order to assess the functionality of these GOS mixtures.

The authors thank the Spanish Ministry of Education and Science (Projects Consolider Ingenio 2010 FUN-C-FOOD 200700063 and AGL2007-62736) and Community of Madrid (project ALIBIRD-CM S-0505/AGR-000153) for financial support. Alejandra Cardelle-Cobas and Nuria Salazar thank their predoctoral FPU and FPI grants, respectively, from the Spanish Ministry of Education and Science. The authors thank Friesland Food Domo for providing them with Vivinal-GOS.

\section{References}

Aider M \& de Halleux D 2007 Isomerization of lactose and lactulose production: a review. Trends in Food Science and Technology 18 356-364

Al-Tamimi MAY, Palframan RJ, Cooper JM, Gibson GR \& Rastall RA 2006 In vitro fermentation of sugar beet arabinan and arabinooligosaccharides by the human gut microflora. Journal of Applied Microbiology 100 407-414

Anthony JC, Merriman TN \& Heimbach JT 2006 90-Day oral (gavage) study in rats with galactooligosaccharides syrup. Food Chemistry and Toxicology 44 819-826

Barreteau H, Delattre C \& Michaud P 2006 Production of oligosaccharides as promising new food additive generation. Food Technology and Biotechnology 44 323-333

Bouhnik Y, Raskine L, Simoneau G, Vicaut E, Neut C, Flourie B, Brouns F \& Bornet FR 2004a The capacity of nondigestible carbohydrates to stimulate fecal bifidobacteria in healthy humans: a double-blind, randomized, placebo-controlled, parallel-group, dose-response relation study. American Journal of Clinical Nutrition 80 1658-1664

Bouhnik Y, Attar A, Joly FA, Riottot M, Dyard F \& Flourie B 2004b Lactulose ingestion increases faecal bifidobacterial counts: a randomised double-blind study in healthy humans. European Journal of Clinical Nutrition $\mathbf{5 8}$ 462-466

Cardelle-Cobas A, Villamiel M, Olano A \& Corzo N 2008a Study of galacto-oligosaccharide formation from lactose using Pectinex-Ultra SP-L. Journal of the Science of Food and Agriculture 88 954-961

Cardelle-Cobas A, Martínez-Villaluenga C, Villamiel M, Olano A \& Corzo N 2008b Synthesis of oligosacchariedes derived from lactulose and Pectinex Ultra SP L. Journal of Agricultural and Food Chemistry $\mathbf{5 6}$ 3328-3333

Chockchaisawasdee S, Athanasopoulos VI, Niranjan K \& Rastall RA 2005 Synthesis of galacto-oligosaccharides from lactose using, $\beta$-galactosidase from Kluyveromyces lactis: Studies on batch and continuous UF membrane fitted bioreactors. Biotechnology and Bioengineering 89 434-443

Chow J 2002 Probiotics and Prebiotics: a brief overview. Journal of Renal Nutrition 12 76-86

Cristl SU, Murgatroyd PR, Gibson GR \& Cummings JH 1992 Production metabolism and excretion of hydrogen in the large intestine. Gastroenterology 102 1269-1277

Cummings JH 1981 Short chain fatty acids in the human colon. Gut 22 763-779

De Preter V, Vanhoutte T, Huys G, Swings J, Rutgeerts P \& Verbeke K 2008 Baseline microbiota activity and influence responses to prebiotic initial bifidobacteria counts dosing in healthy subjects. Alimentary Pharmacology and Therapeutics 27 504-513

Delzenne NM 2003 Oligosaccharides: state of the art. Proceedings of the Nutrition Society 62 177-182

Djouzi Z \& Andrieux C 1997 Compared effects of three oligosaccharides on metabolism of intestinal microflora in rats inoculated with a human faecal flora. British Journal of Nutrition 78 313-324

Dongowski G, Jacobasch G \& SchmiedI D 2005 Structural stability and prebiotic properties of resistant starch type 3 increase bile acid turnover and lower secondary bile acid formation. Journal of Agricultural and Food Chemistry 53 9257-9267

Gibson GR \& Roberfroid MB 1995 Dietary modulation of the human colonic microbiota: introducing the concept of prebiotics. Journal of Nutrition 125 1401-1412

Gueimonde M, Tölkko S, Korpimäki T \& Salminen S 2004 New real-time quantitative PCR procedure for quantification of bifidobacteria in human fecal samples. Applied and Environmental Microbiology 70 4165-4169

Holt SM, Miller-Fosmore CM \& Côté GL 2005 Growth of various intestinal bacteria on alternansucrase-derived oligosaccharides. Letters in Applied Microbiology 40 385-390

Hsu CA, Lee SL, Chou CC 2007 Enzymatic production of galactooligosaccharides by $\beta$-galactosidase from Bifidobacterium longum BCRC 15708. Journal of Agricultural and Food Chemistry $\mathbf{5 5}$ 2225-2230

Kaneko T, Kohmoto T, Kikuchi H, Shiota M, lino, H \& Mitsuoka T 1994 Effects of isomaltooligosaccharides with different degrees of polymerization on human faecal Bifidobacteria. Bioscience Biotechnology and Biochemistry 58 2288-2290

Kaplan H \& Hutkins RW 2000 Fermentation of fructooligosaccharides by lactic acid bacteria and bifidobacteria. Applied and Environmental Microbiology 66 2682-2684

Louis P, Scott KP, Duncan SH \& Flint HJ 2007 Understanding the effects of diet on bacterial metabolism in the large intestine. Journal of Applied Microbiology 102 1197-1208

Macfarlane S \& Macfarlane GT 2003 Regulation of short-chain fatty acid production. Proceedings of the Nutrition Society 62 67-72

Macfarlane S, Macfarlane GT, Cummings JH 2006 Review article: prebiotics in the gastrointestinal tract. Alimentary Pharmacology \& Therapeutics 24 701-714 
Mahoney RR 1998 Galactosyl-oligosaccharide formation during lactose hydrolysis: a review. Food Chemistry 63 147-154

Martínez-Villaluenga C, Cardelle-Cobas A, Corzo N, Olano A \& Villamiel M 2008a Optimization of conditions for galactooligosaccharide synthesis during lactose hydrolysis by $\beta$-galactosidase from Kluyveromyces lactis (Lactozym 3000 L HP G). Food Chemistry 107 258-264

Martínez-Villaluenga C, Cardelle-Cobas, A, Olano A, Corzo N, Villamiel M, Jimeno, ML 2008b Enzymatic synthesis and identification of two trisaccharides produced from lactulose by transgalactosylation. Journal of Agricultural and Food Chemistry 56 557-563

Matteuzzi D, Swennen E, Rossi M, Hartman T \& Lebet V 2004 Prebiotic effects of a wheat germ preparation in human healthy subjects. Food Microbiology 21 119-124

Mendez A \& Olano A 1979 Lactulose. A review of some chemical properties and application in infant nutrition and medicine. Dairy Sciences Abstracts 41 531-535

Onishi N, Yamashiro A \& Yokozeki K 1995 Production of galactooligosaccharide from lactose by Sterignatomyces elviae CBS8119. Applied and Environmental Microbiology 61 4022-4025

Panesar PS, Panesar R, Singh RS, Kennedy JF \& Kumar H 2006 Microbial production, immobilization and applications of $\beta$-galactosidase. Journal of Chemical Technology and Biotechnology 81 530-543

Rycroft CE, Jones MR, Gibson GR, Rastall RA 2001 A comparative in vitro evaluation of the fermentation properties of prebiotic. Journal of Applied Microbiology 91 878-887

Salazar N, Gueimonde M, Hernández-Barranco MH, Ruas-Madiedo P \& de los Reyes-Gavilan CG 2008 Exopolysaccharides produced by intestinal Bifidobacterium strains act as fermentable substrates for human intestinal bacteria. Applied and Environmental Microbiology $\mathbf{7 4}$ 4737-4745

Sanz ML, Gibson GR \& Rastall RA 2005 Influence of disaccharide structure on prebiotic selectivity in vitro. Journal of Agricultural and Food Chemistry 53 5192-5199

Shin HJ \& Yang JW 1998 Enzymatic production of galactoligosacchatides by Bullera singularis $\beta$-galactosidase. Journal of Microbiology and Biotechnology 8 484-489

Splechtna B, Nguyen TH, Steinbock M, Kulbe KD, Lorenz W \& Haltrich D 2006 Production of prebiotic galactooligosacchairdes from lactose using $\beta$-galactosidase from Lactobacillus reuteri. Journal of Agricultural and Food Chemistry $\mathbf{5 4}$ 4999-5006

Tomomatsu H 1994 Health effects of oligosaccharides Food Technology 48 61-65

Topping DL \& Clifton PM 2001 Short-chain fatty acids and human colonic function: roles of resistant starch and nonstarch polysaccharides. Physiological Reviews 81 1031-1064

Tuohy KM, Rouzaud GCM, Brück WM \& Gibson GR 2005 Modulation of the human gut microflora towards improved health using prebioticsAssessment of efficacy. Current Pharmaceutical Design 11 75-90

Vanhoutte T, De Preter V, Brandt E, Verbeke K, Swings J \& Huys G 2006 Molecular monitoring of the fecal microbiota of healthy human subjects during administration of lactulose and Saccharomyces boulardii. Environmental Microbiology 72 5990-5997

Wong JMW, Souza de R, Kendall CWC, Emam A \& Jenkins DJA 2006 Colonic health: fermentation and short chain fatty acids. Journal of Clinical Gastroenterology 40 235-243 